\title{
Families Affected by Huntington's Disease Report Difficulties in Communication, Emotional Involvement, and Problem Solving
}

Celine M.H. Jona ${ }^{\mathrm{a}}$, Izelle Labuschagne ${ }^{\mathrm{a}, \mathrm{b}}$, Emily-Clare Mercieca ${ }^{\mathrm{a}}$, Fiona Fisher ${ }^{\mathrm{c}}$, Cathy Gluyas $^{\mathrm{c}}$, Julie C. Stout ${ }^{\mathrm{a}}$ and Sophie C. Andrews ${ }^{\mathrm{a}, *}$

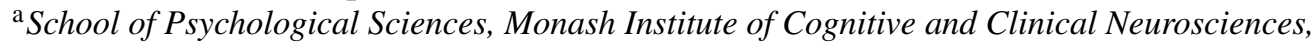
Faculty of Medicine, Nursing and Health Sciences, Monash University, Clayton Campus, VIC, Australia

${ }^{\mathrm{b}}$ Cognition and Emotion Research Centre, School of Psychology, Australian Catholic University,

Fitzroy, Australia

${ }^{\mathrm{c} C a l v a r y}$ Health Care Bethlehem, Caulfield, VIC, Australia

\begin{abstract}
.
Background: Family functioning in Huntington's disease (HD) is known from previous studies to be adversely affected. However, which aspects of family functioning are disrupted is unknown, limiting the empirical basis around which to create supportive interventions.

Objective: The aim of the current study was to assess family functioning in HD families.

Methods: We assessed family functioning in 61 participants (38 HD gene-expanded participants and 23 family members) using the McMaster Family Assessment Device (FAD; Epstein, Baldwin and Bishop, 1983), which provides scores for seven domains of functioning: Problem Solving; Communication; Affective Involvement; Affective Responsiveness; Behavior Control; Roles; and General Family Functioning.

Results: The most commonly reported disrupted domain for HD participants was Affective Involvement, which was reported by $39.5 \%$ of HD participants, followed closely by General Family Functioning (36.8\%). For family members, the most commonly reported dysfunctional domains were Affective Involvement and Communication (both 52.2\%). Furthermore, symptomatic HD participants reported more disruption to Problem Solving than pre-symptomatic HD participants. In terms of agreement between pre-symptomatic and symptomatic HD participants and their family members, all domains showed moderate to very good agreement. However, on average, family members rated Communication as more disrupted than their HD affected family member.

Conclusion: These findings highlight the need to target areas of emotional engagement, communication skills and problem solving in family interventions in HD.
\end{abstract}

Keywords: Affective involvement, communication, family, family functioning, Huntington's disease, problem solving

\footnotetext{
${ }^{*}$ Correspondence to: Dr. Sophie C. Andrews, Monash University, Wellington Rd \& Blackburn Rd, Clayton VIC 3800, Australia. Tel.: +61 3990 20481; E-mail: sophie.andrews@ monash.edu.
} 


\section{INTRODUCTION}

Huntington's disease (HD) is an inherited neurodegenerative disorder that causes neuropsychiatric, cognitive and motor dysfunction. HD is autosomal dominant, so children with an affected parent have a $50 \%$ chance of developing HD [1]. Despite the intergenerational nature of HD, few studies have analysed family functioning in this population. With the high levels of care needed for those with HD and the nature of the symptomology of HD, particularly the early psychiatric and cognitive symptoms [e.g. 2, 3] it is likely that aspects of family functioning are affected in this population.

Although no study has directly assessed the impact of HD on family functioning, two studies have described family functioning in this population. Vamos and colleagues used questionnaires to retrospectively assess family relationships and parenting styles in 40 adults who grew up in a household with HD. These researchers found that on measures of family functioning, participants demonstrated low levels of family cohesion, verbal expressiveness, and higher levels of conflict, all of which fell in the dysfunctional range [4]. In another study, Maxted and colleagues studied seven parent/adult child dyads using qualitative methods to explore experiences and relationships in these HD families [5]. The major themes that emerged included a tendency to protect those members they identified as feeling closest to, while isolating family members who were unwilling to accept the presence of the disease, and the changing of identities by virtue of shifting and adapting of roles within the family.

Although the research to date provides valuable qualitative insights into family functioning associated with HD, a quantitative assessment of family functioning in HD is needed to identify what proportion of families experience disrupted family functioning, and which aspects of family functioning are most affected.

Family functioning refers to the ability of a family to work together effectively, to deal with conflicts, and to fulfil their basic needs [6]. This concept is based upon a family system perspective, which assumes that family members form a complex and interconnected system, and thus the functioning of the family system can be altered by the presence of a terminal illness in one or more family members [6]. According to the McMaster Model, family functioning consists of six independent and clinically relevant elements (Affective Involvement, Affective
Responsiveness, Behavior Control, Communication, Problem Solving, and Roles), along with one General Family Functioning domain [7]. Specifically, Affective Involvement refers to the extent of interest of family members in each other; Affective Responsiveness describes the ability of family members to experience appropriate affect; Behaviour Control relates to the expression and maintenance of behaviour standards; Communication refers to the way family members exchange information; Problem Solving denotes the ability of families to resolve issues that come up; Roles refers to patterns of behaviour for handling basic family functions and whether these functions are clearly and equitably assigned, and lastly General Family Functioning represents the global health/pathology of the family. A quantitative measure of family functioning based on the McMaster Model, is the Family Assessment Device (FAD). The FAD has been widely used in both nonclinical and clinical population, including dementia [8], making it arguably the most extensively researched measure of family functioning to date [9]. The FAD provides includes cut-off scores to differentiate between well-functioning and disrupted family functioning [7, 10]. Although the FAD has not previously been used to assess family functioning in HD, Tremont and colleagues [8] used the FAD to analyse the relationship between family functioning and caregiver burden in families affected by dementia. They identified poorer family functioning especially in the Roles and Communication domains, in families with high burden. These findings provide preliminary insights into how families with chronic diseases function.

Common symptoms in HD during the pre-manifest and early manifest period include psychiatric symptoms such as anxiety, irritability and depression [2], cognitive symptoms such as executive dysfunction and poor emotion recognition [3], and lastly, changes to behaviour and diminished self-awareness [11]. These symptoms are known to affect communication, problem solving and behaviour in individuals with HD, and therefore it is likely that these aspects of family functioning are also affected. In addition, these symptoms are likely to exacerbate stress within HD families more generally.

The aim of the current study was to assess HD family functioning in families using the FAD. Unlike the two previous studies $[4,5]$ of family functioning in $\mathrm{HD}$, we aimed to apply a well-validated and broadly used family assessment measure, which will facilitate our understanding of HD family functioning and 
enable comparisons with the functioning of families with other illnesses. To increase the depth of understanding of family functioning in families affected by $\mathrm{HD}$, we examined family functioning from the perspectives of family members who were premanifest or symptomatic HD, and by their non-genetically atrisk family members. It was proposed that gaining multiple perspectives would enable a more complete picture of how family functioning might be experienced differently based on different roles engendered by being an affected or an unaffected family member.

Given that no previous research has looked at which individuals make up a typical HD family, we firstly aimed to describe a typical family with HD and their five closest members according to the HD individuals. Further, given previous findings from Vamos and colleagues [4] Maxted and colleagues [5], and Tremont and colleagues [8], we hypothesized that Communication, Roles, and Affective Involvement would be the most disrupted aspects of family functioning. Given that with time, symptoms of HD gradually worsen [e.g. 12], we also expected that functioning would be more disrupted in families of symptomatic gene-expanded participants than pre-symptomatic participants. Because people with HD may show reduced insight into their physical and mental changes, significantly worsening with increased symptom severity [11], a secondary aim was to examine the levels of agreement between
HD participants and their close family members' ratings on scores on the FAD. Due to reduced insight, commonly experienced by HD individuals [11], we predicted that we would see fewer family functioning concerns from HD participants compared to family members.

\section{MATERIALS AND METHODS}

\section{Participants}

\section{Sample characteristics}

The sample comprised a total of 61 participants, including 38 people with the HD gene expansion (21 premanifest, 17 with early diagnosis), and 23 nominated close family members who were not at risk for HD. Refer to Fig. 1 for study participation diagram. Participants were recruited through a research volunteer database at Monash University, and advertisements at a local health care provider and a local HD community event.

To be included in the study, participants were required to be at least 18 years of age, and confirmed to have the HD gene expansion via genetic testing. Because the focus of the study was family functioning, we requested all HD gene-expanded participants to nominate one close family member. Twenty-three $(61 \%)$ nominated a family member who participated in the study. To reduce the possibility of sample bias, we did not exclude those unable to nominate a

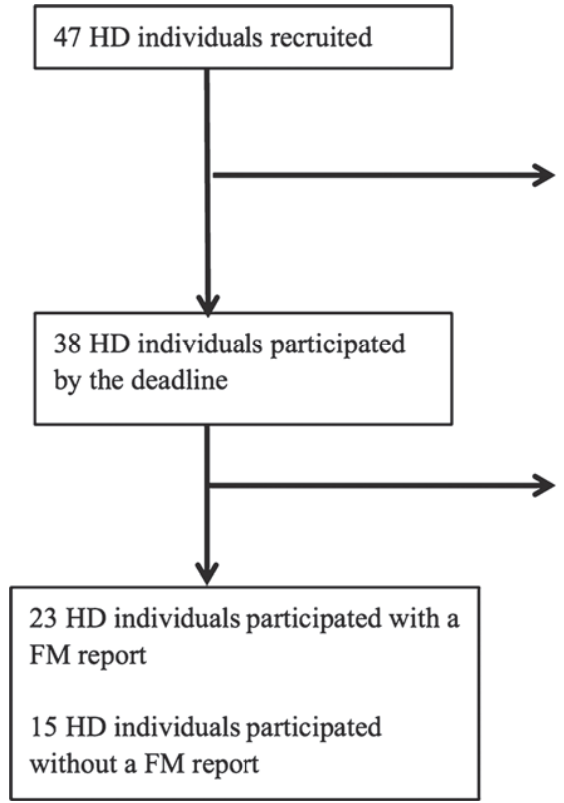

$5 \mathrm{HD}$ individuals were unable to complete the study within the study period

$4 \mathrm{HD}$ individuals started the survey but did not complete it

$9 \mathrm{HD}$ participants were unable to organise their FM to participate within the study period

4 participants were unable to find a FM who was willing to complete the study

2 FMs excluded (one because they were genepositive, and one because the family member was the son of a HD participant)

Fig. 1. Study participation diagram. $\mathrm{HD}=$ Huntington's disease participant, FM= Family Member. 
Table 1

Demographics of HD participants, HD sub-groups and family members

\begin{tabular}{|c|c|c|c|c|}
\hline & $\begin{array}{c}\text { Overall HD } \\
\text { group } \\
(n=38)\end{array}$ & $\begin{array}{c}\text { HD with FM } \\
\text { report } \\
(n=23)\end{array}$ & $\begin{array}{c}\text { FM } \\
(n=23)\end{array}$ & $\begin{array}{c}\text { HD without } \\
\text { FM report } \\
(n=15)\end{array}$ \\
\hline Age years (Mean, SD) & $52.29(10.64)$ & $52.09(10.07)$ & $51.70(11.30)$ & $52.60(11.84)$ \\
\hline Gender (M:F) & $21: 17$ & $15: 8$ & $7: 16$ & $6: 9$ \\
\hline \multicolumn{5}{|c|}{ Highest education $[n(\%)]$} \\
\hline Primary & $1(2.6)$ & $1(4.3)$ & - & - \\
\hline Secondary & $8(21.1)$ & $5(21.7)$ & $2(8.7)$ & $3(20.0)$ \\
\hline Post-sec & $18(47.4)$ & $9(39.1)$ & $8(34.8)$ & $9(60.0)$ \\
\hline University & $11(28.9)$ & $8(34.8)$ & $13(56.5)$ & $3(20.0)$ \\
\hline \multicolumn{5}{|l|}{ Employment [ $n(\%)]$} \\
\hline Full-time & $13(34.2)$ & $8(34.8)$ & $10(43.5)$ & $5(33.3)$ \\
\hline Part-time/Casual & $7(18.4)$ & $3(13.0)$ & $6(26.1)$ & $4(26.7)$ \\
\hline House duties & - & - & $1(4.3)$ & - \\
\hline Retired & $14(36.8)$ & $9(39.0)$ & $6(26.1)$ & $5(33.3)$ \\
\hline Unemployed & $4(10.5)$ & $3(13.0)$ & - & $1(6.7)$ \\
\hline \multicolumn{5}{|c|}{ Relationship status $[n(\%)]$} \\
\hline Married & $27(71.1)$ & $21(91.3)$ & $22(95.7)$ & $6(40.0)^{*}$ \\
\hline Living with partner & $3(7.9)$ & $1(4.3)$ & $1(4.3)$ & $2(13.3)$ \\
\hline Single & $8(21.1)$ & $1(4.3)$ & - & $7(46.7)$ \\
\hline \multicolumn{5}{|c|}{ Household income $[n(\%)]$} \\
\hline$>\$ 25,000$ AUD & $9(23.7)$ & $6(26.1)$ & $4(17.4)$ & $3(20.0)$ \\
\hline$<\$ 50,000$ AUD & $8(21.0)$ & $4(17.3)$ & $2(8.6)$ & $4(26.7)$ \\
\hline$<\$ 100,000$ AUD & $13(34.2)$ & $7(30.4)$ & $8(34.8)$ & $6(40.0)$ \\
\hline$>\$ 100,000$ AUD & $8(21.1)$ & $6(26.1)$ & $9(39.1)$ & $2(13.3)$ \\
\hline
\end{tabular}

Note: $*$ indicates significantly lower scores compared to the other groups. $\mathrm{HD}=$ Huntington's disease; $\mathrm{FM}=$ family. member.

family member participant. All family members were life-partners of the HD participants. Demographic information for all participants is displayed in Table 1. Participants were compensated for their time and ethical approval was granted by Monash University and Calvary Health Care Bethlehem Human Research Ethics Committees.

\section{Procedure}

The study, including informed consent, was completed as an online survey which required participants to have access to a device with internet connection. The survey consisted of general demographic and familial structure questions, after which the Family Assessment Device (FAD, [7]) was completed. Participants were emailed a link to the survey, and the online system permitted participants to save their work and return to the survey when convenient.

\section{Family Assessment Device}

To assess family functioning, we used the original McMaster Family Assessment Device (FAD, [7]), which is a 53-item self-report measure scored on a four-point scale (strongly disagree to strongly agree).
Items on this scale are categorized into seven domains as shown in Table 2 . The FAD has been validated with both families seeking treatment for family functioning and those not seeking treatment [9]. To evaluate the significance of levels of endorsements of the items, Miller and colleagues developed cut-off scores to distinguish "healthy" and "disrupted" functioning families [10]. Recently these cut-off scores have been validated in a community sample by showing that all domains of functioning (apart from Roles in singleparent households), show elevations in help-seeking families compared to non-help seeking families [9]. Thus, this evidence indicates that the cut-off scores [10] are a valid way to identify those families with intact or disrupted family functioning.

\section{Statistical Analyses}

All analyses (alpha level $=0.05$ ) were conducted using IBM SPSS statistics (version: 22.0). Preliminary analyses were conducted on participant characteristics. For the FAD, cut-off scores from Miller and colleagues [10] were applied to FAD subscale scores to determine the percentage of participants reporting disrupted family functioning. To compare HD and family member groups on 
Table 2

HD scores on the Family Assessment Device

\begin{tabular}{|c|c|c|c|c|c|c|}
\hline & \multirow{2}{*}{$\begin{array}{c}\text { HD Total } \\
n=38 \\
M(S D)\end{array}$} & \multirow{2}{*}{$\begin{array}{c}\text { HD with FM } \\
n=23 \\
M(S D)\end{array}$} & \multirow{2}{*}{$\begin{array}{c}\text { FM } \\
n=23 \\
M(S D)\end{array}$} & \multirow{2}{*}{$\begin{array}{c}\text { Cut off Scores } \\
\text { (Miller et al. [10]) }\end{array}$} & \multicolumn{2}{|c|}{ Families above cut off $(\%)$} \\
\hline & & & & & $\overline{\text { HD overall }}$ & FM \\
\hline Problem Solving & $1.88(0.44)$ & $1.87(0.50)$ & $1.93(0.48)$ & 2.20 & 31.6 & 39.1 \\
\hline Communication & $2.06(0.35)$ & $2.02(0.40)$ & $2.24 *(0.56)$ & 2.20 & 28.9 & 52.2 \\
\hline Roles & $2.21(0.33)$ & $2.13(0.31)$ & $2.16(0.34)$ & 2.30 & 28.9 & 26.1 \\
\hline Affective Responsiveness & $2.01(0.51)$ & $1.97(0.56)$ & $2.04(0.54)$ & 2.20 & 23.7 & 39.1 \\
\hline Affective Involvement & $1.99(0.45)$ & $1.95(0.46)$ & $2.03(0.49)$ & 2.10 & 39.5 & 52.2 \\
\hline Behavior Control & $1.81(0.42)$ & $1.81(0.38)$ & $1.71(0.38)$ & 1.90 & 31.6 & 26.1 \\
\hline General Functioning & $1.77(0.42)$ & $1.69(0.45)$ & $1.76(0.45)$ & 2.00 & 36.8 & 26.1 \\
\hline
\end{tabular}

Note. * Indicates mean is above the Miller and colleagues cut off for dissatisfied family functioning. HD = Huntington's disease participants; $\mathrm{FM}=$ Family Members .

FAD ratings, we used paired samples $t$-tests. Levels of agreement between HD and family member participants were assessed using intraclass correlation coefficients [13]. Pearson's Chi Squared tests was used to determine whether there was a greater proportion of FAD ratings above the cut off when HD participants were symptomatic versus not yet symptomatic.

\section{RESULTS}

\section{Preliminary analyses}

To determine whether HD participants differed depending on whether or not they had a family member willing to participate in the study, we used Pearson Chi Squared. The only difference between HD geneexpanded participants with and without a family member participating was relationship status. Specifically, a higher proportion of those with a family member participant were in an intimate partnership compared to those without a family member participant $\left(X^{2}(2, N=38)=12.02, p=0.002\right)$. Please refer to Table 1 .

\section{Description of typical HD family make-up}

For our first study aim, the HD participant group described who made up their close family members. HD participants described their families as comprising of an average of four close family members. These close family members were siblings (33.7\%), children (30.9\%), parents (15.8\%), partners $(12.2 \%)$, aunts $(2.3 \%)$, cousins $(1.7 \%)$, friends $(1.7 \%)$, uncles $1.2 \%$, grandparents $(0.6 \%)$, grandchildren $(0.6 \%)$, and nieces $(0.6 \%)$. Respondents also reported that the majority of these close family members were also people with whom they lived.

\section{FAD results}

Scores on the FAD and percentages of participants reaching the cut-offs for disruption for a given domain are shown in Table 2. Across the seven subscales, the HD participant group (both presymptomatic and symptomatic) participants reported disrupted functioning ranging from $39.5 \%$ (Affective Involvement) to $23.7 \%$ (Affective Responsiveness), whereas family members reported disrupted functioning ranging from 52.2\% (Affective Involvement and Communication) to $26.1 \%$ (Roles, Behaviour Control, and General Functioning all at 26.1\%). Although no subscales differed significantly between groups, there was a trend for family members to indicate poorer functioning compared to the HD gene-expanded participants for Communication $(t(23)=2.05, p=0.05)$, with a medium effect size $(d=0.47)$. Family members and HD gene-expanded respondents showed moderate to excellent levels of agreement across domains (for results and effect sizes please see Table 3 ).

Comparison of FAD subscores within the HD participants group indicated that symptomatic respondents reported higher levels of disruption in Problem Solving compared with pre-symptomatic respondents, $X^{2}(1, \mathrm{~N}=38)=10.57, p=0.002$, with a large effect size $(\phi=-0.53)$, see Fig. 2 . For the remainder of the domains, we did not see a difference between symptomatic and premanifest participant ratings (all $p s>0.13$ ). In contrast, ratings of family members did not show systematic differences based on whether their close other HD gene-expanded participant was presymptomatic or symptomatic (all $p s>0.21$ ), see Fig. 3 .

\section{DISCUSSION}

Of the seven domains of family functioning in the FAD, Affective Involvement, that is, family 
Table 3

Comparisons between HD and Family Member scores on Family Assessment Device

\begin{tabular}{lccccc}
\hline & $\begin{array}{c}\text { HD vs. FM } \\
t(95 \% \text { CI })^{1}\end{array}$ & $\begin{array}{c}\text { HD vs. FM } \\
(p \text {-value })^{1}\end{array}$ & Cohen's $d$ & $\begin{array}{c}\text { Intraclass } \\
\text { Correlation }\end{array}$ & $\begin{array}{c}\text { ICC category } \\
\text { Problem Solving }\end{array}$ \\
Communication & $2.02(-0.316,0.107)$ & 0.318 & 0.22 & 0.66 & Good \\
Roles & $0.53(-0.452,0.003)$ & 0.053 & 0.47 & 0.55 & Moderate \\
Affective Responsiveness & $0.54(-0.314,0.183)$ & 0.604 & 0.12 & 0.61 & Good \\
Affective Involvement & $0.68(-0.110,0.154)$ & 0.592 & 0.12 & 0.63 & Good \\
Behavior Control & $1.75(-0.061,0.232)$ & 0.094 & 0.16 & 0.82 & Moderate \\
General Functioning $n=23$ & $0.81(-0.259,0.114)$ & 0.430 & 0.16 & 0.71 & Excellent \\
\hline
\end{tabular}

Note. ${ }^{*} p<0.05$. HD $=$ Huntington's disease participant FM $=$ Family Member. ${ }^{1}$ Paired samples $t$-test.

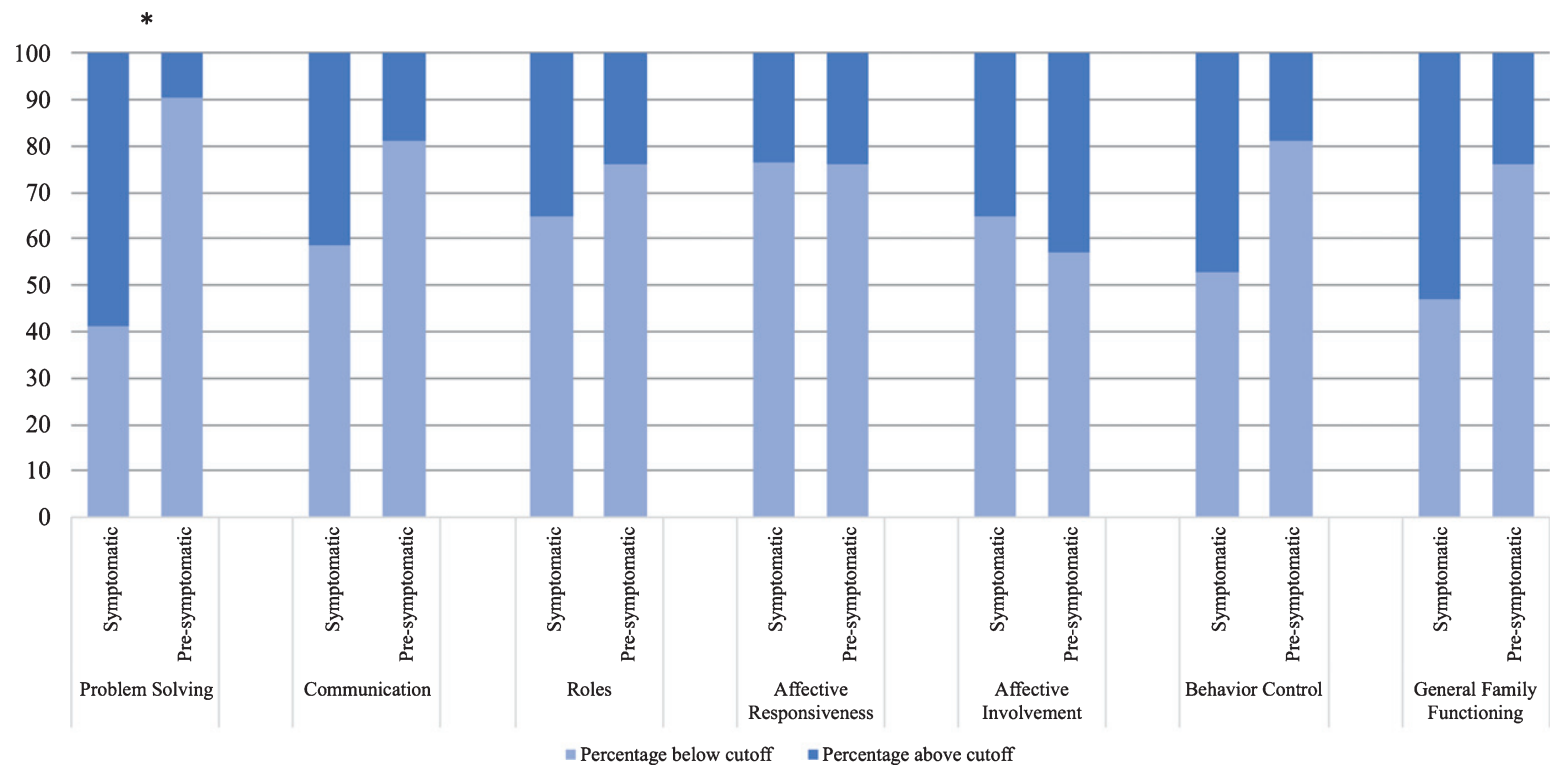

Fig. 2. Proportion of Huntington's disease participants reporting disrupted family functioning on Family Assessment Device subscales, by symptom status. Note. ${ }^{*}=p<0.05$; cut off score for each subscale is shown in Table 2.

members' interest and involvement in the emotional experience of each other, was identified most frequently as disrupted by both HD gene-expanded participants and their family members. Previous studies report reduced expressiveness [4] and a closed off emotional nature of family members to one another [5]. It might be that some HD families experience difficulties expressing and being receptive to others' emotions, which reduces the emotional involvement family members have with one another.

Over half of the family members also identified the FAD domain of Communication, the exchange of information between members, as commonly disrupted within their families, but this issue was less commonly reported within the HD group. Differences in perceived communication difficulties within HD families have been reported previously by professional carers, HD family members, pre-symptomatic and early stage HD individuals, as reported in
Hartelius and colleagues [14]. In this study, all participants identified changes to communication, but the nature of these changes differed between the groups. The family members and carers focused on reporting changes in speech, comprehension of language, an absence of depth in conversation and need for adjustments in communication. HD participants instead focused on the increased energy and concentration required for communication [14]. Some of these communication changes are likely caused by the cognitive changes associated with HD, and the ratings discrepancy between gene-expanded participants and their family members on the Communication (and Affective Involvement) domains may be due to reduced insight in the gene-expanded participants. Our findings extend previous studies of communication in HD to suggest that disrupted communication in HD has an impact on family functioning. Finally, our findings regarding communication is also consistent with 


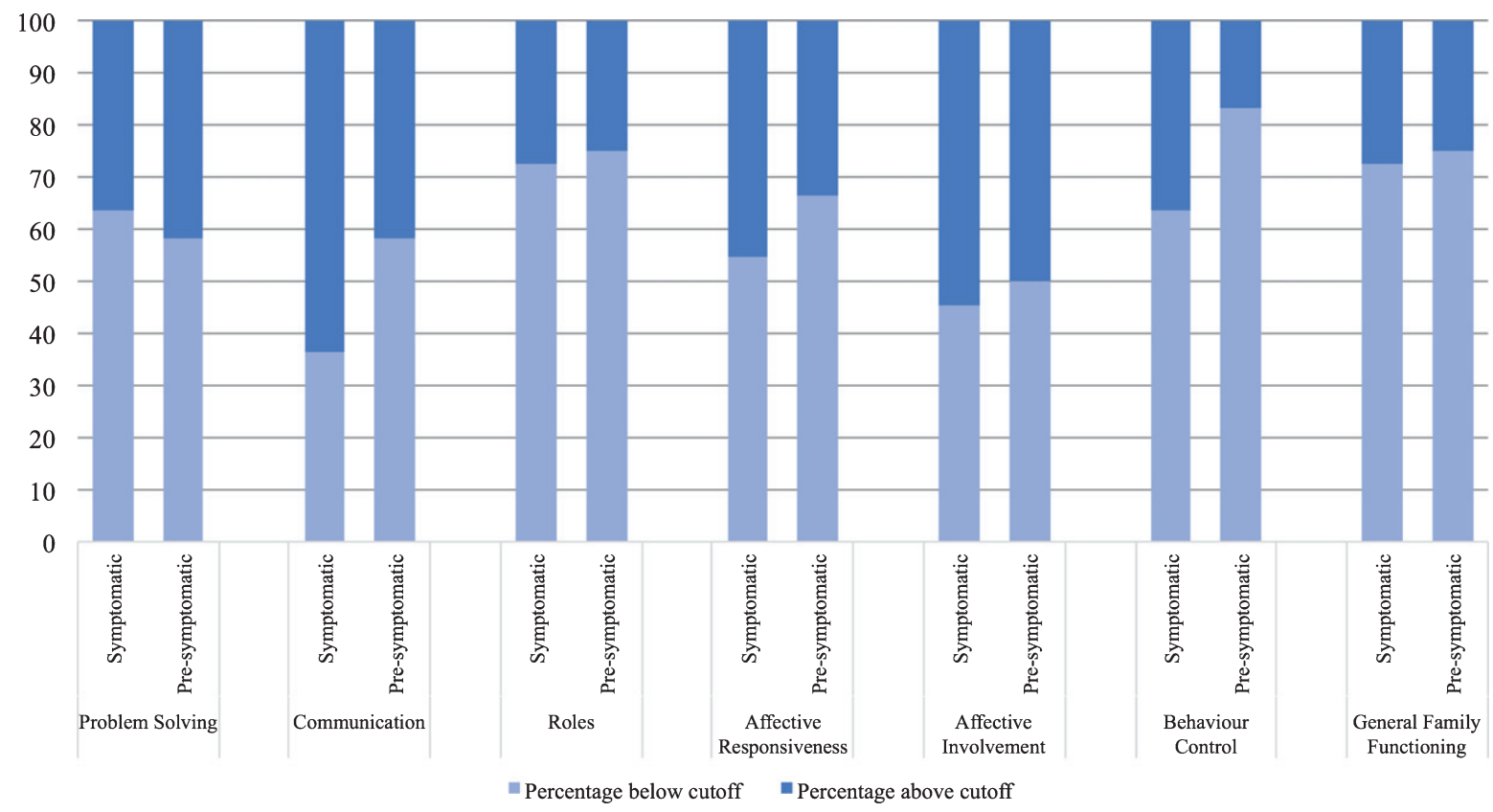

Fig. 3. Proportion of Family Member participants reporting disrupted family functioning on Family Assessment Device subscales, by symptom status of HD participant. Note. Cut off score for each subscale is shown in Table 2.

Tremont and colleagues [8], who found the FAD subdomain of communication was commonly disrupted within families affected by dementia.

Our finding that $39.1 \%$ of family members and more than half of the symptomatic participants (58.8\%) identified Problem Solving as disrupted within their families suggests that this is a significant issue amongst all families, but is most commonly identified by symptomatic HD participants. Cognitive impairment is a common symptom of HD [15], and when it occurs family members often assist with activities of daily living and take on more decision-making responsibilities [16, 17]. One possible explanation for why HD participants are reporting more issues in Problem Solving upon symptom onset, therefore, is that family members may consult less with their HD affected family member when making decisions and solving problems, particularly if the symptomatic HD family member is experiencing cognitive impairment. The nature of collaborative problem-solving may change within the family, therefore, and result in a reduction in consultation when solving problems within symptomatic HD families. If this is confirmed by future research, we suggest that families can work on enhancing their communication with their family unit, to combat the perception of reduced problem solving abilities and enhance feelings of involvement.
Although a considerable proportion of HD families reported dysfunction across the domains (between $23.7 \%$ and $39.5 \%$ of HD participants and between $26.1 \%$ and $52.2 \%$ of family members scored above suggested cut-offs for dysfunction), our results suggest that a large proportion of our sample was generally content with their family functioning. Our results contrast with those of Vamos and colleagues, who reported that the majority of HD families in their study scored in the dysfunctional range for levels of expressiveness, cohesion and conflict, all indicative of suboptimal family functioning [4]. Vamos et al. included only families with a symptomatic HD family member, whereas our study also included pre-symptomatic participants. Thus, it might be that families with symptomatic family members may have different family issues to those who are pre-symptomatic. Our study also contrasts with the findings of Maxted and colleagues, who conducted qualitative identified several prominent themes including the changing and adaptation of roles within parent/adult child dyads [5]. In contrast to our findings, Rothing and colleagues observed major role shifts including parentification (children taking over their parent's role within the family) in HD families [17]. We have two possible explanations for these differences. First, the Roles domain in the FAD does not directly assess role shifts like paren- 
tification, but rather measures patterns of behaviour for handling basic family functions and whether these functions are clearly and equitably assigned. Therefore, parentification may be experienced by our participants but not assessed by our measure. Alternatively, given that the majority of our participants were pre-symptomatic or early HD, it might be that role shifts do not occur until later in the disease, and thus not experienced by the families assessed in the current study. This should be clarified in future HD family functioning research, by using a measure that includes role shifts relevant to HD such as parentification. Given the lack of previous research family functioning in $\mathrm{HD}$, it is also useful to look at this construct in other chronic illnesses such as dementia. Studies assessing family functioning in dementia, also report contrasting results to our study i.e. these families report poorer family functioning especially in Communication and Roles [8]. However, again while studies of other dementias include only symptomatic participants, our study also included pre-symptomatic participants. It might be that during the later stages of disease, families report more disruption to aspects of family functioning compared to families with a presymptomatic or early symptomatic family member and highlights the need to assess family functioning in later stage-HD families.

Given the dearth of research in this area, and thus subject to replication in future studies, we were encouraged to find that a high percentage of families are functioning well (i.e., scores do not indicate disruption) in comparison to the FAD published cutoffs. Similarly, other than Communication we found close agreement within families on family functioning. These findings are encouraging and positive. However, a limitation of the current study is that we had access to too few symptomatic HD participants to explore how family functioning varied in relation to disease progression and other clinical factors such as cognitive function and neuropsychiatric symptoms, which would be a useful addition to current studies to help identify what characteristics are associated with higher risk of particular difficulties in family function. It is also important to bear in mind that in our sample, most participants were gainfully employed and had high levels of education. With disease progression, activities of daily functioning, especially employment become less common [18]. Our results are therefore unlikely to generalize to families in which the person with HD is in a more progressed disease stage. Nonetheless, given our results, a sys- tematic examination of the positive aspects of family functioning may illuminate treatment opportunities when difficulties within HD families do arise. Strategies such as acceptance, emotional support, planning [20] and loyalty [21] are important for successful HD partnerships, and can be relevant for family functioning interventions. With a better understanding of these identified strategies, family interventions can be tailored to suit HD families. Furthermore, early intervention may be indicated, prior to onset of HD symptoms that may impact on their capacity to engage in this therapeutic work.

Our study may be limited in that the sample of HD families who volunteer for research might not represent the general population of HD families. Our HD sample was relatively small, most were gainfully employed, were on average in their early $50 \mathrm{~s}$, many were in a relationship, and all were living in a family setting. Further, given that we recruited our family members through our HD participants, our sample will likely have missed family members that the HD participants were unwilling to include, which could lead to a bias in our sample to families who were functioning better. For future studies, it would be beneficial to include families with well-established symptomatic disease to understand how family function differs with severity of illness. Promisingly, our results also indicate that the majority of these families with pre-symptomatic and in early disease staged HD, are functioning well. Future research could examine what factors contribute to positive family functioning. Finally, the use of control groups tailored to reveal how families with HD differ from other families affected by chronic illness, or from 'healthy' families with no chronic illnesses, would add an important perspective to understanding family function in HD.

Overall, our findings indicated that both HD geneexpanded participants and their family members identify reduced emotional involvement within families, and furthermore HD family members also commonly identify communication issues. People with manifest HD in particular also indicated that they felt less included in matters of problem solving. These findings are relevant for HD clinicians, who could consider working with families around strategies for improved emotional engagement, communication and problem solving.

\section{CONFLICT OF INTEREST}

The authors have no conflict of interest to report. 


\section{REFERENCES}

[1] Richards F, Taylor S. Social work and genetic testing: Ethical issues encountered in predictive testing for Huntington disease. Aust Soc Work. 1997;50(4):61-7. doi:10.1080/03124079708415747

[2] Martinez-Horta S, Perez-Perez J, van Duijn E, FernandezBobadilla R, Carceller M, Pagonabarraga J, PascualSedano B, Campolongo A, Ruiz-Idiago J, Sampedro F, Landwehrmeyer GB, Spanish REGISTRY investigators of the European Huntington's Disease Network, Kulisevsky J. Neuropsychiatric symptoms are very common in premanifest and early stage Huntington's Disease. Parkinsonism Relat Disord. 2016;25:58-64. doi:10.1016/j.parkreldis. 2016.02.008

[3] Papoutsi M, Labuschagne I, Tabrizi SJ, Stout JC. The cognitive burden in Huntington's disease: Pathology, phenotype, and mechanisms of compensation. Mov Disord. 2014;29(5):673-83. doi:10.1002/mds.25864

[4] Vamos M, Hambridge J, Edwards M, Conaghan J. The impact of Huntington's disease on family life. Psychosomatics. 2007;48(5):400-4. doi:10.1176/appi.psy.48.5.400

[5] Maxted C, Simpson J, Weatherhead S. An exploration of the experience of Huntington's disease in family dyads: An interpretative phenomenological analysis. J Genet Couns. 2014;23(3):339-49. doi:10.1007/s10897-013-9666-3

[6] Staccini L, Tomba E, Grandi S, Keitner GI. The evaluation of family functioning by the family assessment device: A systematic review of studies in adult clinical populations. Fam Process. 2015;54(1):94-115. doi:10.1111/famp.12098

[7] Epstein NB, Baldwin LM, Bishop DS. The McMaster Family Assessment Device. J Marital Fam Theory. 1983;9(2): 171-80. doi:10.1111/j.1752-0606.1985.tb00028.x

[8] Tremont G, Davis JD, Bishop DS. Unique contribution of family functioning in caregivers of patients with mild to moderate dementia. Dement Geriatr Cogn Disord. 2006;21(3):170-4. doi:10.1159/000090699

[9] Mansfield AK, Keitner GI, Dealy J. The family assessment device: An update. Fam Process. 2015;54(1):82-93. doi:10.1111/famp.12080

[10] Miller IW, Bishop DS, Epstein NB, Keitner GI. The McMaster Family Assessment Device: Reliability and validity. J Marital Fam Theory. 1985;11(4):345-56. doi:10.1111/ j.1752-0606.1985.tb00028.x

[11] Sitek EJ, Thompson JC, Craufurd D, Snowden JS. Unawareness of deficits in Huntington's disease. J Huntingtons Dis. 2014;3(2):125-35. doi:10.3233/JHD-140109
[12] Folstein SE, Franz ML, Jensen BA, Chase GA, Folstein MF. Conduct disorder and affective disorder among the offspring of patients with Huntington's disease. Psychol Med. 1983;13(1):45-52. doi:10.1017/S0033291700050054

[13] Fleiss JL, Levin B, Paik MC. Statistical Methods for Rates and Proportions. 3rd ed. ed. Hoboken: John Wiley \& Sons; 2003.

[14] Hartelius L, Jonsson M, Rickeberg A, Laakso K. Communication and Huntington's disease: Qualitative interviews and focus groups with persons with Huntington's disease, family members, and carers. Int J Lang Commun Disord. 2010;45(3):381-93. doi:10.3109/13682820903105145

[15] Dumas EM, Say MJ, Jones R, Labuschagne I, O'Regan AM, Hart EP, van den Bogaard SJ, Queller S, Justo D, Coleman A, Dar Santos RC, Durr A, Leavitt BR, Tabrizi SJ, Roos RA, Stout JC. Visual Working Memory Impairment in Premanifest Gene-Carriers and Early Huntington's Disease. J Huntingtons Dis. 2012;1(1):97-106. doi:10.3233/JHD2012-120010

[16] Williams JK, Skirton H, Barnette JJ, Paulsen JS. Family carer personal concerns in Huntington disease. $\mathrm{J}$ Adv Nurs. 2012;68(1):137-46. doi:10.1111/j.1365-2648.2011. 05727.x

[17] Rothing M, Malterud K, Frich JC. Caregiver roles in families affected by Huntington's disease: A qualitative interview study. Scand J Caring Sci. 2014;28(4):700-5. doi:10.1111/scs.12098

[18] Beglinger LJ, O'Rourke JJ, Wang C, Langbehn DR, Duff K, Paulsen JS, Huntington Study Group I. Earliest functional declines in Huntington disease. Psychiatry Res. 2010;178(2):414-8. doi:10.1016/j.psychres.2010.04.030

[19] Bogod NM, Mateer CA, MacDonald SW. Self-awareness after traumatic brain injury: A comparison of measures and their relationship to executive functions. J Int Neuropsychol Soc. 2003;9(3):450-8. doi:10.10170S1355617703930104

[20] Downing NR, Williams JK, Leserman AL, Paulsen JS. Couples' coping in prodromal Huntington disease: A mixed methods study. J Genet Couns. 2012;21:662-70. doi:10.1007/s10897-012-9480-3

[21] Richards F. Couples' experiences of predictive testing and living with the risk or reality of Huntington disease: A qualitative study. Am J Med Genet A. 2004;126A(2):170-82. doi:10.1002/ajmg.a.20583 